\title{
Modeling and mesoscopic damage constitutive relation of brittle short-fiber-reinforced composites *
}

\author{
LIU Hongqiu (刘洪秋), LIANG Naigang（梁乃刚） \\ (LNM, Institute of Mechanics, Chinese Academy of Sciences, Beijing 100080 , China) \\ and XIA Mengfen (夏蒙禁) \\ (Department of Physics, Center for Nonlinear Scrence, Peking Uraversiiy, Beijing 0087 !, Clina)
}

Received Apri1 5, 1999

\begin{abstract}
rimed at britile compusies roinforced by randomly distributed short-fibers with a relatively large aspect ratio, stiffness modulus and sirength, a mesoscopic material model was proposed. Based on the statistical description, damage n:ch ${ }^{\prime}$ :sms, damage-induced anisotropy, damage rate effect and stress redistribution, the constitutive relation were derived. By taking glass fiber reinforced polypropylene polymers as an example, the effect of initial orientation distribution of fibers, damage-induced anisotropy, and damage-rate effect on macro-behaviors of composites were quantitatively analyzed. The theoretical predictions compared favorably with the experimental results.
\end{abstract}

Keywords: short-fiber reinforced composites, mesoscopic material model, damage constitutive theory, damage-induced anisotropy, damage-rate effect.

Short-fiber-reinforced composites (SFRCs) are increasingly applied because they are cost-effective and display superior mechanical properties. Some damage constitutive equations derived for this kind of material are based on the self-consistent scheme and equivalent inclusion method. An approximate extension of Eshelby's method proposed by Mori and Tanaka ${ }^{[1]}$ has been widely adopted for fiber reinforced composites recently. However, most of the material models and damage constitutive equations are still not good enough for composites with a relatively dense, large aspect ratio and randomly distributed fibers. Liang and Bergan ${ }^{[2]}$ introduced the fiber-reinforcing and sliding mechanisms into a material model, so a polycrystalline metal model and mesoscopic elastoplastic constitutive relation were proposed $^{[3]}$; and the physical mechanism of the deformation-induced anisotropy was embodied ${ }^{[4]}$; the mesoscopic description of the fiber-reinforcing mechanism was recently applied to multiphase fiber-reinforced composite materials ${ }^{[5]}$; those suggest a new way to develop a damage theory for SRFCs.

Continuous Damage Mechanies for homogeneous material was developed on the basis of a damage concept proposed by Kachanov ${ }^{[6]}$. By using the intrinsic theory of thermodynamics, Rousselier ${ }^{[7]}$ brought the damage variable into the framework of Continuum Mechanics. Later, Murakami ${ }^{[8]}$ extended the isotropic damage constitutive equation ${ }^{[9]}$ into an anisotropy theory. By using statistical description about micro-crack initiation as decohesion, Liu and Liang ${ }^{[10]}$ proposed an anisotropic damage model to simulate the damage behavior of polycrystalline metals. From a parallel bundle model for long-fiber-reinforced composites, Krajeinovic ${ }^{[11]}$ suggested that the damage evolution is attributable to

* Project supported by the National Natural Science Foundation of China (Grant Nos. 19872065 and 19732060) and the Chinese Academy of Sciences. 
the probability distribution of rupture strength of fibers which made a great contribution for the damage theory of SFRCs.

In contrast to continuum mechanics, the main difficulty in establishing the damage theory of SFRCs lies in position and orientation heterogeneity of fibers, damage sensitivity to the initial-defects and damage pattern in matrix, damage anisotropy depending on loading-path and deformation-history, interaction between matrix and fibers, as well as coupling between differently oriented fibers. Therefore, a new idea is needed to develop a damage theory for SFRCs. There are still challenging problems for SFRCs including how to construct a material model with microstructure, how to describe the damage evolution laws of the fiber, matrix, and interface, and how to briage a ga: between mesoscopic physical components and macroscopic behavior of composites ${ }^{[: 2]}$.

Owing to the complexity of material and the irinitaiion of experimental data, three basic assumptions were made in the present najer to simplify the mathemaitical description of the constitutive equations for engineering application. The first is how the local strains relate to the overall strain in subsec. 1. . The second is about ile stability of the statistical mean value of parameters for a representative elenient volume (REV) large enough in size in subsec. 1.2. The third concerns the unimportance of the interaction between differently oriented fibers in subsection 2.3.

Based on those assumptions and in view of the microstructural characteristics and physical properties of constituents, a meso material model was constructed first, followed by the derivation of a damage-rate-dependent constitutive equation with consideration of the probability density of fiber rupture, orientation distribution density, and stress-redistribution during fiber-breaking. An example was given for glass fiber reinforced polypropylene polymers. The effect of initial orientation, damage-induced anisotropy, and damage-rate effect on macro-behaviors of the material was quantitatively analyzed. The theoretical predictions compared favorably with the experimental results, which demonstrate the predictability of the present model. Some conclusions and discussion are given in the last section .

\section{Material modeling}

\subsection{The heterogeneity of local stress-strain}

SFRCs consist of matrix and randomly distributed short-fibers. Due to the position and orientation heterogeneity, the local strain and stress in the matrix and reinforcers relate to the macro strain in a complicated manner. In the present study, it is assumed that local strain rate in the matrix and fibers is dependent on their positions, but at each position it remains proportional to the macro strain rate before damage takes place. Therefore, the heterogeneity of local strain rates can be described by using of heterogeneity factors.

Let $\dot{\boldsymbol{E}}_{m}$ and $\dot{\tilde{\varepsilon}}_{m}$ denote the local strain rates of the matrix and fiber, respectively, $\boldsymbol{P}=\boldsymbol{l} \circledast \boldsymbol{l}$ denote an orientation tensor of fibers in a unit vector $\boldsymbol{l}$, and $\dot{\boldsymbol{E}}$ denote a macro-strain rate tensor. Thus, the above assumption can be stated as

$$
\begin{aligned}
& \dot{\tilde{\boldsymbol{E}}}_{m}=\tilde{\boldsymbol{c}}_{m}: \dot{\boldsymbol{E}}, \\
& \dot{\tilde{\varepsilon}}_{f}=\tilde{c}_{f} \dot{\varepsilon} \text { where } \dot{\boldsymbol{\varepsilon}}=\boldsymbol{P}: \dot{\boldsymbol{E}},
\end{aligned}
$$

where $\bar{c}_{m}$ and $\bar{c}_{f}$ are heterogeneity factors of the matrix and fibers, " $\sim$ " means they are functions of 
location. Due to the interaction of micro-components, $\tilde{c}_{m}$ and $\tilde{c}_{f}$ vary generally with macro-deformation $\boldsymbol{E}$.

As a primary study, attention is limited to an infinitesimal strain during an isothermal process, and mechanical properties of constituents are incremental linear. Let $\dot{\boldsymbol{S}}_{m}$ and $\widetilde{\boldsymbol{K}}_{m}$ denote the stress rate and transient stiffness tensor of the matrix, respectively, and $\dot{\sigma}_{f}$ and $\tilde{k}_{f}$ denote the stress rate and the transient tangent modulus of the fiber, respectively. The local incremental stress-strain relations of the matrix and the fiber can be written as

$$
\begin{aligned}
& \dot{\boldsymbol{S}}_{m}=\widetilde{\boldsymbol{K}}_{m}: \dot{\tilde{\boldsymbol{E}}}_{m}, \quad . \\
& \dot{\sigma}_{f}=\tilde{k}_{f} \dot{\varepsilon}_{f}
\end{aligned}
$$

\subsection{Modeling of unidircetional-sh}

First we con:idet the composites reinforced by short fibers randomly located in the matrix but oriented in only ore direction. The total deformation power of REV is equal to the sum of ones contributed by the matrix and fibers, i.e.

$$
\boldsymbol{S}: \dot{\boldsymbol{E}}=\int_{v_{m}} \tilde{\boldsymbol{S}}_{m}: \dot{\widetilde{\boldsymbol{E}}}_{m} \mathrm{~d} v+\int_{v_{f}} \tilde{\sigma}_{f} \dot{\tilde{\varepsilon}}_{f} \mathrm{~d} v=\left\{\int_{v_{m}} \tilde{\boldsymbol{S}}_{m}: \tilde{\boldsymbol{c}}_{m} \mathrm{~d} v+\int_{v_{f}} \tilde{c}_{f} \tilde{\sigma}_{f} \mathrm{~d} v \boldsymbol{P}\right\}: \dot{\boldsymbol{E}} .
$$

In the isothermal and elastic process, the total power is identical to the rate of specific Helmholtz' free energy, and the macro stress of the composites will be

$$
\boldsymbol{S}=\int_{v_{m}} \tilde{\boldsymbol{S}}_{m}: \tilde{\boldsymbol{c}}_{m} \mathrm{~d} v+\int_{v_{f}} \tilde{c}_{f} \tilde{\sigma}_{f} \mathrm{~d} v \boldsymbol{P} .
$$

Thus, the total stress is shared by the matrix and fibers together. The macro stress rate can be derived as

$$
\dot{\boldsymbol{S}}=\int_{v_{m}}\left(\dot{\tilde{\boldsymbol{S}}}_{m}: \tilde{\boldsymbol{c}}_{m}+\tilde{\boldsymbol{S}}_{m}: \dot{\tilde{\boldsymbol{c}}}_{m}\right) \mathrm{d} v+\int_{v_{f}}\left(\tilde{c}_{f} \dot{\sigma}_{f}+\dot{\bar{c}}_{f} \tilde{\sigma}_{f}\right) \mathrm{d} v \boldsymbol{P} .
$$

Substituting eqs. (1), (2) into eqs. (3), (4) and then into eq. (7) yield

$$
\dot{\boldsymbol{S}}=\left(v_{m} \overline{\boldsymbol{K}}_{m}+v_{f} \overline{\boldsymbol{K}}_{f}\right): \dot{\boldsymbol{E}},
$$

where

$$
\begin{gathered}
\overline{\boldsymbol{K}}_{m}=\frac{1}{v_{m}} \int_{v_{m}}\left[\tilde{\boldsymbol{c}}_{m}: \widetilde{\boldsymbol{K}}_{m}: \tilde{\boldsymbol{c}}_{m}+\frac{1}{2}\left(\tilde{\boldsymbol{S}}_{m}: \frac{\mathrm{d} \tilde{\boldsymbol{c}}_{m}}{\mathrm{~d} \boldsymbol{E}}+\frac{\mathrm{d} \tilde{\boldsymbol{c}}_{m}}{\mathrm{~d} \boldsymbol{E}}: \overline{\boldsymbol{S}}_{m}\right)\right] \mathrm{d} v, \\
\overline{\boldsymbol{K}}_{f}=\bar{k}_{f} \boldsymbol{P} \otimes \boldsymbol{P} \text { where } \bar{k}_{f}=\frac{1}{v_{f}} \int_{v_{f}}\left(\tilde{c}_{f}^{2} \tilde{k}_{f}+\tilde{\sigma}_{f} \frac{\mathrm{d} \tilde{c}_{f}}{\mathrm{~d} \varepsilon}\right) \mathrm{d} v
\end{gathered}
$$

are macro stiffness tensors contributed by the matrix and fibers, respectively, $v_{m}$ and $v_{f}$ are volume fractions of the matrix and fibers, respectively. Eqs. (8)-(10) show an incremental constitutive relation of heterogeneously unidirectional SFRCs in an isothermal and non-damage process.

Therefore, the unidirectional SFRCs can be modeled with the continuous matrix with $\overline{\boldsymbol{K}}_{m}$ and the fiber-bundle with $\bar{k}_{f}$. The matrix and fiber-bundle satisfy

$$
\begin{aligned}
& \dot{\boldsymbol{S}}_{m}=\overline{\boldsymbol{K}}_{m}: \dot{\boldsymbol{E}}, \\
& \dot{\sigma}_{f}=\bar{k}_{f} \dot{\varepsilon}_{f} \text { where } \dot{\varepsilon}_{f}=\boldsymbol{P}: \dot{\boldsymbol{E}},
\end{aligned}
$$

respectively. The average stiffness of the matrix and fiber-bundle represents the statistical characteris- 
tics of the random distribution. Hence, the model can simulate the macro mechanical behavior of practical composites. The variation of the average stiffness with deformation can be determined statistically for specific materials. It should be noted that unlike long-fiber reinforced composites, REV is much larger than the length of fiber itself, and the aspect ratio, stiffness modulus and strength are greater. The property of fiber-bundles depends not only on the fiber property but also on the heterogeneity of the fiber distribution. Therefore, the fiber-bundle is equivalent to the long fibers with a stiffness $\bar{k}_{f}$. Based on the minor interaction between differently oriented fibers, the above material model can be extended to randomly oriented SRFCs (see subsec . 2.3). In the next section, we will formulate the damage constitutive equation on the basis of the niodel with unidirectional short-fibers. Without causing any confusion, the bars above the average sriffness tensors will be omitted.

\section{Damage constitutive relation}

2.1 Statistical expression and thencodynamic reiation of damage variables

Demage of SFRCis generaliy includes deterioration of the matrix, matrix-fiber interface debonding, fiber-treaking and their interactions. Damage of the matrix and interface destroys the force-transfer path, while fiber damage causes a concentration of defects and stress in the matrix and interface. Therefore, no matter what kinds of damage take place, the load-bearing capacity of constituents will decrease so as to result in a progressive deterioration of the average mechanical property of composites. It is too difficult to describe every detail of such a complicated damage process. Thus, the complicated damage is attributed to the matrix deterioration and fiber-bundle damage due to fiber-breaking and interface-debonding.

According to Kachanov's damage definition ${ }^{[6]}$, the average stresses of the matrix and fiber-bundle satisfy

$$
\begin{aligned}
& \boldsymbol{S}_{m}=\left(1-D_{m}\right) \boldsymbol{S}_{m}^{(\mathrm{ef})}, \\
& \sigma_{f}=\left(1-D_{f}\right) \sigma_{f}^{(\mathrm{ef})},
\end{aligned}
$$

where $D_{m}$ is Kachanov's damage factor of the degraded matrix, and $D_{f}$, a percentage of the damaged fiber-bundle due to fiber-breaking and interface-debonding. Therefore, effective stresses, $S_{m}^{(e f)}$ and $\sigma_{f}^{(\text {ef })}$ obey the ordinary stress-strain laws :

$$
\begin{aligned}
& \dot{\boldsymbol{S}}_{m}^{(\mathrm{ef})}=\boldsymbol{K}_{m}: \dot{\boldsymbol{E}}, \\
& \dot{\sigma}_{f}^{(\mathrm{ef})}=k_{f} \dot{\varepsilon}_{f},
\end{aligned}
$$

where $\boldsymbol{K}_{m}$ is the average stiffness tensor of the matrix, and $k_{f}$, the average stiffness modulus of fiberbundles. Statistically, let $\psi\left(\varepsilon_{f c}\right)$ denote a probability distribution density of the broken or debonding strain $\varepsilon_{f c}$ of fibers. It should satisfy the normalizing condition

$$
\int_{\varepsilon_{c r}}^{\infty} \psi\left(\varepsilon_{f c}\right) \mathrm{d} \varepsilon_{f c}=1 .
$$

Thus, $D_{f}$ can be calculated by the following equation

$$
D_{f}\left(\varepsilon_{f}\right)=\int_{\varepsilon_{\sigma}}^{\varepsilon_{f}} \psi\left(\varepsilon_{f_{c}}\right) \mathrm{d} \varepsilon_{f c},
$$

where $\varepsilon_{c r}$ is the critical broken strain of the weakest fibers, and $\varepsilon_{f}$ is the fiber accumulated strain . Generally, $\varepsilon_{f}$ should be the maximum strain reached during a deformation history. Therefore, the 
damage evolution of the fiber-bundle will be determined by the probability distribution density and the maximum strain of the fiber-bundle.

For an isothermal and infinitesimal-strain process, independent state variables are the strain tensor $\boldsymbol{E}$ and the damage vector $\boldsymbol{D}=\left\{D_{m}, D_{f}\right\}$. If $f$ denotes the specific Helmholtz free energy, the generalized Gibbs' relation ${ }^{[13]}$ can be expressed as

$$
\rho_{0} \dot{f}=\boldsymbol{S}: \dot{\boldsymbol{E}}-\boldsymbol{\eta} \cdot \dot{\boldsymbol{D}},
$$

where $\rho_{0}$ is mass density. The quantities conjugate to $\boldsymbol{E}$ and $\boldsymbol{D}$ are the stress tensor $\boldsymbol{S}$ and the generalized thermodynamic force $\boldsymbol{\eta}=\left\{\eta_{m}, \eta_{f}\right\}$, respectively. $\boldsymbol{S}$ and $\boldsymbol{\eta}$ are functions of $\boldsymbol{E}$ and $\boldsymbol{D}$. Since $\boldsymbol{\eta}=\boldsymbol{\eta}(\boldsymbol{E}, \boldsymbol{D})$, we can write $\boldsymbol{D}=\boldsymbol{D}(\boldsymbol{E}, \boldsymbol{\eta})$. The damiage evolution cal thus be expressed as

$$
\dot{D}=\frac{\partial D}{\partial E}: \dot{E}+\frac{\partial D}{\partial} \cdot \dot{\eta} \cdot
$$

During a quasi-static process in which ihe internal variable $\boldsymbol{\eta}$ is fully controlled by the macro strain, i.e. $\boldsymbol{\eta}=\boldsymbol{\eta}(\boldsymbol{E})$, the a'sove equavion can be further expressed as

$$
\dot{\boldsymbol{D}}=\left\{\frac{\partial \boldsymbol{D}}{\partial \boldsymbol{E}}+\frac{\partial \boldsymbol{D}}{\partial \boldsymbol{\eta}} \frac{\mathrm{d} \boldsymbol{\eta}}{\mathrm{d} \boldsymbol{E}}\right\}: \dot{\boldsymbol{E}}=\frac{\mathrm{d} \boldsymbol{D}}{\mathrm{d} \boldsymbol{E}}: \dot{\boldsymbol{E}} .
$$

In this case, the damage rate is proportional to the strain rate, and the proportional coefficient depends on $\frac{\mathrm{d} \boldsymbol{D}}{\mathrm{d} \boldsymbol{E}}$. Evidently, $\frac{\mathrm{d} \boldsymbol{D}}{\mathrm{d} \boldsymbol{E}}$ is material-dependent. For fiber-bundles, it can be obtained from eq. $(18)$, i.e.

$$
\frac{\mathrm{d} D_{f}}{\mathrm{~d} \boldsymbol{E}}=\frac{\mathrm{d} D_{f}}{\mathrm{~d} \varepsilon_{f}^{h}} \frac{\mathrm{d} \varepsilon_{f}^{h}}{\mathrm{~d} \boldsymbol{E}}=\psi\left(\varepsilon_{f c}\right) \boldsymbol{P}
$$

\subsection{Damage-rate effect and the damage constitutive equation}

In the event of damage, the total deformation power of REV is equal to the sum of contributions of the matrix and fiber-bundle. By using eqs. (13) and (14), the total power is

$$
\boldsymbol{S}: \dot{\boldsymbol{E}}=v_{m}\left(1-D_{m}\right) \boldsymbol{S}_{m}^{(\text {ef })}: \dot{\boldsymbol{E}}+v_{f}\left(1-D_{f}\right) \sigma_{f}^{(\mathrm{ef})} \boldsymbol{P}: \dot{\boldsymbol{E}} .
$$

It derives

$$
\boldsymbol{S}=v_{m}\left(1-D_{m}\right) \boldsymbol{S}_{m}^{(\mathrm{ef})}+v_{f}\left(1-D_{f}\right) \sigma_{f}^{(\mathrm{ef})} \boldsymbol{P} ;
$$

that is, the total stress of composites during damage is still shared by the effective matrix and fiberbundle together. And the total stress rate should be

$$
\dot{\boldsymbol{S}}=v_{m}\left\{\left(1-D_{m}\right) \dot{\boldsymbol{S}}_{m}^{(\text {ef })}-\dot{D}_{m} \boldsymbol{S}_{m}^{(\text {eff })}\right\}+v_{f}\left\{\left(1-D_{f}\right) \dot{\sigma}_{f}^{(\text {ef })}-\dot{D}_{f} \sigma_{f}^{(\text {ef })}\right\} \boldsymbol{P} \text {. }
$$

It can be seen that the total stress rate is affected by the damage-rate effect as well as the state of the damage. In view of the thermodynamic considerations, the damage rate can be expressed by the macro strain rate. Substituting eqs. (15), (16) and eq. (21) into eq. (25) yields

$$
\dot{\boldsymbol{S}}=\left\{v_{m} \boldsymbol{K}_{m}^{(\mathrm{ef})}+v_{f} \boldsymbol{K}_{f}^{(\mathrm{ef})}\right\}: \dot{\boldsymbol{E}},
$$

where the fourth rank stiffness tensors

$$
\begin{aligned}
& \boldsymbol{K}_{m}^{(\text {ef })}=\left(1-D_{m}\right) \boldsymbol{K}_{m}-\frac{1}{2}\left(\boldsymbol{S}_{m}^{(\mathrm{ef})} \otimes \frac{\mathrm{d} D_{m}}{\mathrm{~d} \boldsymbol{E}}+\frac{\mathrm{d} D_{m}}{\mathrm{~d} \boldsymbol{E}} \otimes \boldsymbol{S}_{m}^{(\text {ef })}\right), \\
& \boldsymbol{K}_{f}^{(\text {ef })}=k_{f}^{(\text {ef })} \boldsymbol{P} \otimes \boldsymbol{P} \quad \text { where } k_{f}^{(\text {ef })}=k_{f}\left(1-D_{f}\right)-\sigma_{f}^{(\text {ef })} \frac{\mathrm{d} D_{f}}{\mathrm{~d} \varepsilon_{f}}
\end{aligned}
$$

are contributed from the effective matrix and fiber-bundle, respectively. $\boldsymbol{K}_{f}^{(\text {ef })}$ embodies the effect of 
damage and damage rate of the fiber-bundle on the macro stiffness tensor. From eqs. (27) and (28), the stiffness reduction can also be divided into two parts. One can be expressed by damage-state variables, and the other, by the damage rate. The influence of the later on the stiffness tensor is called damage-rate effect.

Here, there is no damage healing and developing as $\left|\varepsilon_{f}\right|$ decreases, i.e. $\dot{D} \equiv 0$ during $\left|\varepsilon_{f}\right|$ decreases. The effective stiffness modulus of the fiber-bundle can be stated as

$$
k_{f}^{(\mathrm{e})}= \begin{cases}k_{f}\left(1-D_{f}\right)-\frac{1}{1-D_{f}} \sigma_{f} \frac{\mathrm{d} D_{f}}{\mathrm{~d} \varepsilon_{f}}, & \left|\varepsilon_{f}\right| \geqslant \max \left(\left|\varepsilon_{f}^{h}\right|,\left|\varepsilon_{c r}\right|\right) \text { and } \varepsilon_{f} c \varepsilon_{f}>0, \\ k_{f}\left(1-D_{f}\right), & \left|\varepsilon_{f}\right|<\max \left(\left|\varepsilon_{f}^{h}\right|,\left|\varepsilon_{c r}\right| \text { ) or } \varepsilon_{f} \mathrm{~d} \varepsilon_{f} \leqslant 0 .\right.\end{cases}
$$

During a process without damage-growing, the effective stiffness tensor of the matrix is

$$
\boldsymbol{K}_{m}^{(e f)}=\left(i-D_{n}\right) \boldsymbol{K}_{m},
$$

which is the same as the previous continuum danage theory. Therefore, when the damage-rate effect has little influcine on the effective stiffness tensor, eq. (30) is an approximate formulation of the effective siffrress tensor. The damage-growing law of the matrix is material-dependent. An example will be given in the next section.

To sum up, eqs. (26)-(28) are the incremental meso-damage constitutive equations of unidirectional SFRCs. The effective stiffness tensor depends on the damage-rate effect as well as the danage state. And they indicate also that damage-rate effect will play a very significant role with the increase of damage and/or stress. It will eventually become a dominant factor to cause additional effective stress rate in material ${ }^{[11]}$, even resulting in damage evolution induced catastrophe ${ }^{[12]}$.

\subsection{The damage constitutive relation of 3-D oriented SFRCs}

Generally speaking, short-fibers in composites are randomly oriented in 3-D space. Statistically, one can introduce a probability density $\rho(\Omega)$ to describe the orientation distribution of fiber-bundles, where $\Omega$ is the solid angle in a domain corresponding to a hemispherical surface. Thus, composites reinforced by 3-D oriented short-fibers can also be modeled with the matrix and fiber-bundles with an orientation distribution density $\rho$. Tips of unit vectors of all fiber-bundles form a hemisphere. The orientation distribution density of fiber-bundles is the description of the orientation structure of composites. Usually, the orientation structure of long-fiber composites is much simpler than that of SFRCs.

According to eq. (28), the contribution of fiber-bundles to the macro stiffness tensor depends on $\boldsymbol{P}, k_{f}^{(\text {ef })}, v_{f}$. In view of the slight interaction of oriented fibers in REV, the total power contributed by all oriented fibers can be expressed by superposition, and the stiffness tensor of 3-D fibers becomes

$$
\boldsymbol{K}_{f}^{(\mathrm{e})}=\int_{\Omega} \rho k_{f}^{(\mathrm{ef})} \boldsymbol{P} \otimes \boldsymbol{P} \mathrm{d} \Omega,
$$

where $k_{f}^{(\text {ef })}$ satisfies eq. (29). The orientation distribution density $\rho$ satisfies the normalizing condition

$$
\int_{\Omega} \rho \mathrm{d} \Omega=\int_{0}^{2 \pi} \int_{0}^{\frac{\pi}{2}} \rho \sin \theta \mathrm{d} \theta \mathrm{d} \phi=1,
$$

where $\theta$ and $\phi$ are spherical coordinates. For isotropic distribution, $\rho=1 / 2 \pi$. The anisotropic distribution may result from the manufacturing process and damage. Changing the equation on the left side of eq. (28) into eq. (31) gives the damage constitutive equation for 3-D oriented SFRCs. 


\section{Damage analysis of glass fiber-reinforced polypropylene composites}

Damage of composites in the present paper is attributed to the matrix deterioration and fiber-bundle damage due to fiber-breaking and interface-debonding. The following will take glass fiber-reinforced polypropylene composites as an example, and the damage evolution law will be discussed. By making use of the present material model and general form of the damage constitutive equation, the synthetic effect of constituents on the macro-mechanical behavior of composites can be investigated, and the theoretical results can be compared to experimental data.

\subsection{Damage evolution law of the fiber-bundle}

Statistically, for SFRCs, the normalized Weibull distribucion with a cut-of: $\vdots 9$ commonly accepted, i.e.

$$
\psi\left(\varepsilon_{f c}\right)= \begin{cases}0, & \left|\varepsilon_{f c}\right|<\left|\varepsilon_{c r}\right|, \\ \frac{\lambda_{f} m}{\varepsilon_{0}}\left\{\frac{\varepsilon_{f c}}{\varepsilon_{0}}\right\}^{\left(n_{f}-1\right)} \exp \left\{-\lambda_{f}\left[\left(\frac{\varepsilon_{f c}}{\varepsilon_{0}}\right)^{n_{f}}-\left(\frac{\varepsilon_{c r}}{\varepsilon_{0}}\right)^{n_{f}}\right]\right\}, \quad\left|\varepsilon_{f c}\right| \geqslant\left|\varepsilon_{c r}\right| .\end{cases}
$$

By integrating the above equation, the damage evolution law can be obtained as

$$
D_{f}\left(\varepsilon_{f}\right)= \begin{cases}0, & \left|\varepsilon_{f}\right|<\left|\varepsilon_{c r}\right|, \\ 1-\exp \left\{-\lambda_{f}\left[\left(\frac{\varepsilon_{f}}{\varepsilon_{0}}\right)^{n_{f}}-\left(\frac{\varepsilon_{c r}}{\varepsilon_{0}}\right)^{n_{f}}\right]\right\}, & \left|\varepsilon_{f}\right| \geqslant\left|\varepsilon_{c r}\right|,\end{cases}
$$

where $\lambda_{f}$ is a damage coefficient of the fiber-bundle, $n_{f}$, the Weibull exponent, and $\varepsilon_{0}$, a dimensionless material constant. Consequently, the damage rate can be expressed as

$$
\frac{\mathrm{d} D_{f}}{\mathrm{~d} \varepsilon_{f}}= \begin{cases}0, & \left|\varepsilon_{f}\right|<\max \left(\left|\varepsilon_{f}^{h}\right|,\left|\varepsilon_{c r}\right|\right) \text { or } \varepsilon_{f} \mathrm{~d} \varepsilon_{f} \leqslant 0, \\ \frac{\lambda_{f} n_{f}}{\varepsilon_{0}}\left(1-D_{f}\right)\left\{\frac{\varepsilon_{f}}{\varepsilon_{0}}\right\}^{\left(n_{f}-1\right)}, & \left|\varepsilon_{f}\right| \geqslant \max \left(\left|\varepsilon_{f}^{h}\right|,\left|\varepsilon_{c r}\right|\right) \text { and } \varepsilon_{f} \mathrm{~d} \varepsilon_{f}>0 .\end{cases}
$$

Therefore, the damage-rate effect is dependent on the loading-path and damage history .

For the damage-growing process $\left(\dot{D}_{f}>0\right)$, the damage critical condition corresponding to $n_{f}=$ 2 can be expressed as

$$
\frac{1}{2} k_{f} \varepsilon_{f}^{2}-\frac{1}{2} k_{f} \varepsilon_{c r}^{2} \geqslant 0,
$$

which is analogous to the well known Griffith's criterion for crack propagation.

\subsection{Damage evolution law of the matrix}

Experimental results show that matrix damage is closely related to the degradation of fiber-bundles in the glass fiber-reinforced polypropylene composites. In the following damage analysis, let matrix damage be proportional to the average damage of fiber-bundles, i.e.

$$
D_{m}=\lambda_{m} v_{f}^{n} \bar{D}_{f} \text { where } \bar{D}_{f}=\int_{\Omega} \rho D_{f} \mathrm{~d} \Omega,
$$

where $\lambda_{m}$ is a matrix-damage coefficient, and $n_{m}$, a damage exponent of the matrix with respect to fiber volume fraction. The damage evolution or damage-rate tensor of the matrix can be expressed as

$$
\frac{\mathrm{d} D_{m}}{\mathrm{~d} \boldsymbol{E}}=\lambda_{m} v_{f}^{n_{m}} \int_{\Omega_{d}} \rho \frac{\mathrm{d} D_{f}}{\mathrm{~d} \varepsilon_{f}} \boldsymbol{P} \mathrm{d} \Omega,
$$

where $\Omega_{d g}$ is an orientation domain or a set satisfying damage-growing conditions of fiber-bundles, i.e. 


$$
\Omega_{d g}=\left\{\mathrm{d} \Omega ;\left|\varepsilon_{f}\right| \geqslant \max \left(\left|\varepsilon_{f}^{h}\right|,\left|\varepsilon_{c r}\right|\right) \text { and } \varepsilon_{f} \mathrm{~d} \varepsilon_{f}>0\right\} .
$$

Substituting eqs. (35) and (38) into eqs. (26), (27) and (31) yields the specific form of the damage constitutive equations for glass fiber-reinforced polypropylene composites.

\subsection{Mechanical parameters of the matrix and fiber-bundles}

Based on the experimental investigation of Thomason and Vlug ${ }^{[14]}$, the reinforcers in laminates are basically oriented randomly in a plane, so the fiber distribution density can be expressed as

$$
\rho=\frac{1}{\pi} \delta\left(\theta-\frac{\pi}{2}\right) \text {, }
$$

where $\delta$ is Dirac $\delta$-function. For an initially isotropic distribution, $\rho=1,2 \pi$. In the foliowing examples, attention is focused on the anisotropy effect, so the heterogeneity of local strain rates in the matrix and fiber-bundles is neglected and it is caiken that $\tilde{c}_{m}=\tilde{c}_{n^{2}} \gg<I$ and $\tilde{c}_{m}=\tilde{c}_{f} \equiv 1$, where $I \triangleright \triangleleft$ $\boldsymbol{I}$ is the fourth rank unit tensur. In this case', the eiastic stiffness tensor of composites can be fully determined by phisical constants of the matrix and fibers, that is to say, $E_{m}=1.6 \mathrm{GPa}, \nu_{m}=0.4$, and $k_{f}=76 \mathrm{CF}$. Otiver materiai constants can be calibrated according to a part of the experimental results as $\epsilon_{c r}=0.01, \varepsilon_{0}=0.038, \lambda_{f}=21.0, n_{f}=4.0, \lambda_{m}=12.0, n_{m}=0.5$, respectively. In the present study, 360 discrete orientations are selected for fibers oriented in a plane, and 1440 , for fibers oriented in 3-D space. The physical properties of composites depend on the distribution density and material constants of the matrix and fibers.

\subsection{Overall behavior, damage-induced anisotropy and damage-rate effect}

Figure 1 gives a comparison of predicted results with experimental data for glass fiber-reinforced polypropylene laminates. Fig. 1(a) and (b) show that the tensile modulus and strength of laminates are almost proportional to fiber volume fraction. However, the failure strain of laminates has a strongly nonlinear relation with fiber volume fraction (see fig. 1(c)). The overall property of laminates becomes orthogonally anisotropic induced by damage. The strain softening results from the damage-rate effect and the interaction between the matrix and fiber-bundle damage. All of the predicted tensile modulus and strength as well as the failure strain of the comporsites match the experimental results obtained by Thomason et al. ${ }^{[14,15]}$ As lack of the intrinsic stiffness of the tensile instrument, the strain corresponding to $E_{11}=-2 E_{m}$ is approximately taken as a failure limit in the present numerical analyses.
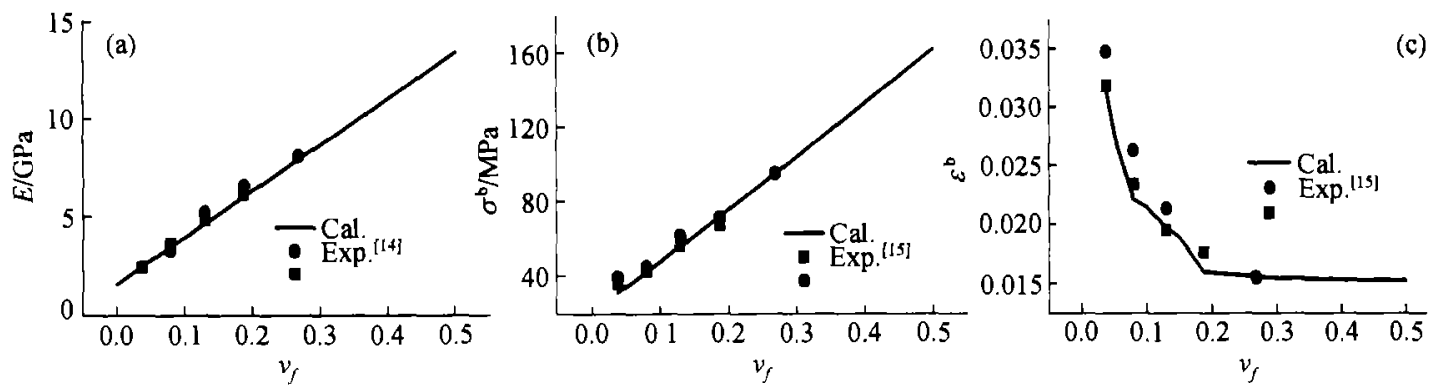

Fig. 1. Variations of tensile modulus, strength and failure strain of laminates with different fiber volume fraction, where marks are experimental data from refs. [14] and [15]. (a) Tensile modulus vs. fiber volume fraction; (b) tensile strength vs. fiber volume fraction; (c) tensile failure strain vs. fiber volume fraction. 
A tensile curve embodies a whole process from an elastic stage to the damage evolution till rupture. A uniaxial extension will cause overall anisotropy of composites, but the damage-induced anisotropy can hardly be measured by the simple extension. The present model is able to analyze the overall anisotropy by examining the variation of the stiffness tensor. In the following numerical analysis, the initially isotropic composite material reinforced by $30 \%$ fibers is taken as an example. Fig. 2 shows variations of engineering constants $E_{11} / E_{m} \& E_{22} / E_{m}\left(=E_{33} / E_{m}\right), G_{12} / G_{m}\left(=G_{13} / G_{m}\right) \&$ $G_{23} / G_{m}$ and $\nu_{12}$ with the tensile strain. Evidently, they all decrease with the increase of the tensile strain, while $E_{11}$ decreases most rapidly. Bumps in curves result from the damagn-ratio effect. The discrepancy between $E_{11} \& E_{22}$ and $G_{12} \& G_{23}$ indicates that the injticiliy isotiopic composite is
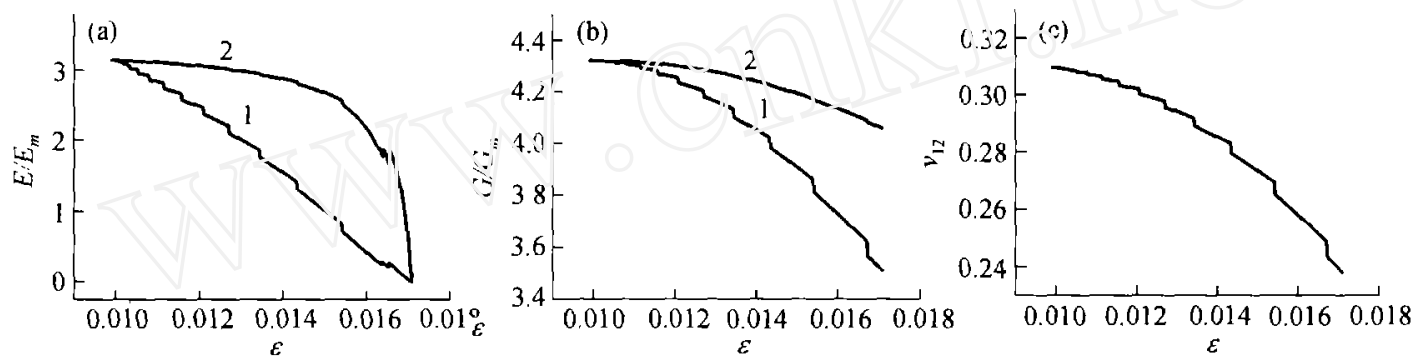

Fig. 2. Variatıons of engineenng constants of sotropic composites with tensile strain. (a) $E_{11} / E_{m}$ (line 1) and $E_{22} / E_{m}$ (line 2) vs. tensile strain; (b) $G_{12} / G_{m}$ (line 1) and $G_{23} / G_{m}$ (line 2) vs. tensile strain; (c) $\nu_{12}$ vs. tensile strain.

changed into a transversely isotropic material. The damage-induced anisotropy grows stronger and stronger with the damage evolution.

\subsection{The effect of initial orientation distribution on overall behavior}

With the wide application of injection moulding technique of SFRCs, the effect of the orientation concentration on the overall behavior becomes more and more significant. The orientation distribution affects the overall performance as well as the stiffness tensor. For injection moulding, the orientation distribution density can be approximately described as a cosine-type function, i.e.

$$
\rho=\frac{1}{2 \pi}(n+1) \cos ^{n} \theta, n=0,1,2, \cdots,
$$

where $x_{1}$-axis is the injection direction, $\theta$, an inclination angle with $x_{1}$-axis. $n=0$ corresponds to an isotropic distribution, while $n \rightarrow \infty$ tends to unidirectional composites. Thus, $n$ can be considered as the measurement of fiber orientation concentration so as to be used to analyze the influence of fiber orientation on the overall behavior of composites.

Generally, the influence of fiber orientation concentration on the overall performance is very complicated, especially in the case of the interaction of initial anisotropy and damage-induced anisotropy. For composites with the cosine-type distribution, its initial property is transversely isotropic. Fig. 3 shows variations of tensile and shear moduli with the tensile strain for composites with $n=$ $0,1,3,5$. The initial tensile modulus $E_{11}$ increases strongly with the increase of $n$, but its degrade velocity with damage is the fastest. This shows the reason for high strength and low toughness for composites with a strongly preferred orientation. However, the initially transverse tensile modulus $E_{22}$ and shear modulus $G_{23}$ decrease with the increase of $n$ and damage. The variation of $E_{22}$ with $n$ and dam- 
age is much more complicated than others, and it is strongly nonlinear. It can be seen that the preferred orientation of fibers offers the potential for enhancing some mechanical performance of composites but suffers the degradation of other properties. Therefore, it is of great significance to control fiber-orientation for optimizing the performance of composites.
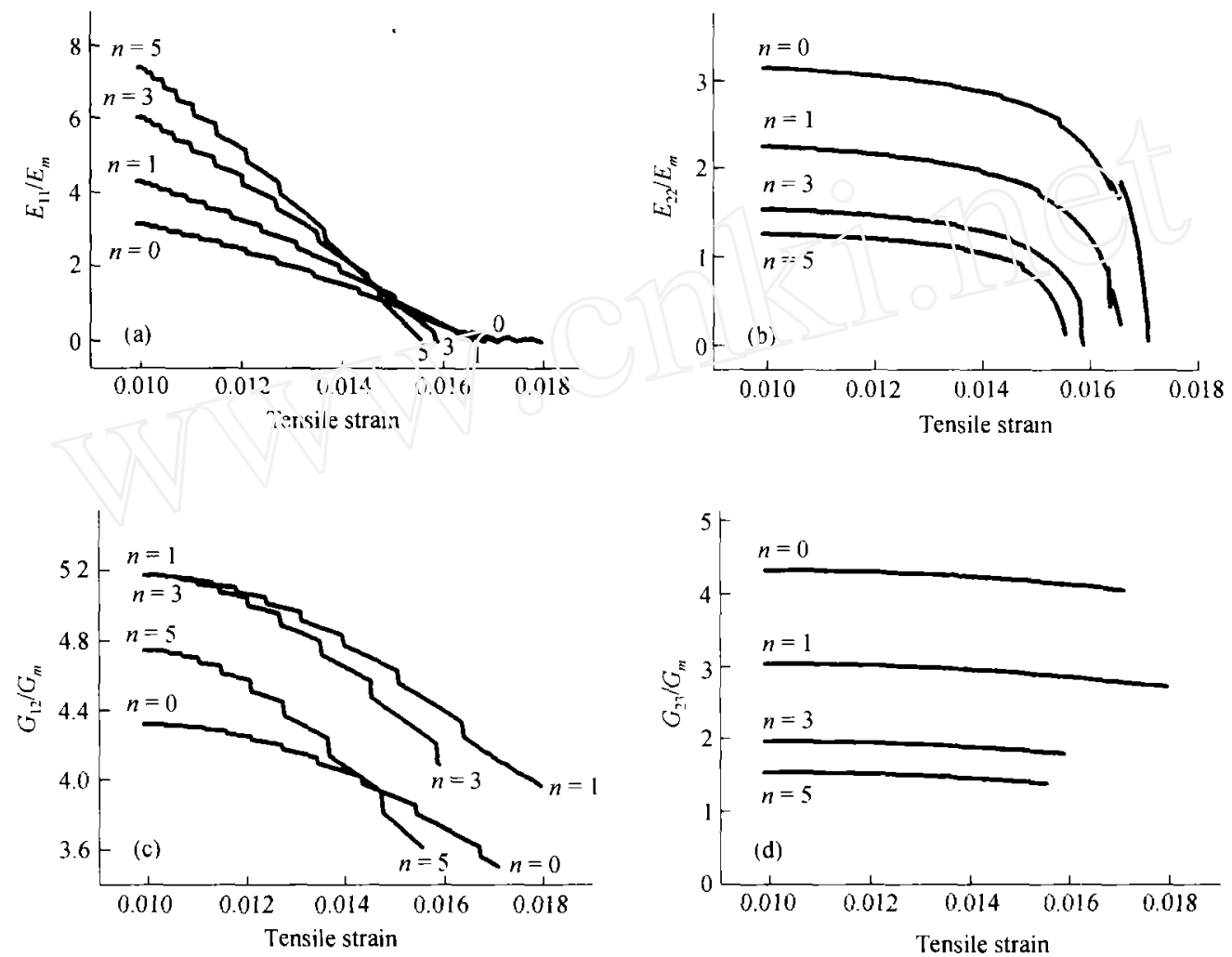

Fig. 3. Variations of tensile and shear moduli with tensile strain for composites with $n=0,1,3,5$. (a) $E_{11} / E_{m}$ vs . tensile strain; (b) $E_{22} / E_{m}$ vs. tensile strain; (c) $G_{12} / G_{m}$ vs. tensile strain; (d) $G_{23} / G_{m}$ vs. tensile strain.

\section{Conclusion and discussion}

A meso physical-mechanism-based material model is constructed for SFRCs which can be applied to simulate and predict the overall mechanical behavior with damage. The constitutive equation is derived with the initial and damage-induced anisotropy as well as the damage-rate effect taken into account. It is flexible and convenient for application to SFRCs with different fiber orientation distributions, including isotropic, plane and cosine-type distributions mentioned in numerical analyses.

The initial anisotropy caused by a preferred orientation and damage-induced anisotropy significantly influence the mechanical property of SFRCs. The damage-induced anisotropy depends on the loading path and deformation history.

The damage-rate effect vitally affects the ability to support the additional load increments of composites. During a stress-controlled loading process, the damage-rate effect leads to the strength failure or damage evolution induced catastrophe far earlier than complete damage. And during a strain-controlled loading process, the damage-rate effect results in the strain softening of composites. 
Of course, the actual microstructure of SFRCs is much more complicated than the present material model. The present work is only a primary attempt. We hope the present material model will be helpful.to serve as a bridge linking the microscopic material parameters of the composites to the macro mechanical behavior for engineêring applịcations and optimum design of SFRCs.

\section{References}

1 Mori, T., Tanaka, K., Average stress in matrix and average energy of materials with misfitting inclusions, /ct $\approx$ Metallurgica et Materialia, 1973, 21: 571 .

2 Liang, N.G., Bergan, P. G., A multi-dimension composite model of elasto-plastic continua under ncn-propcrtiora! loading condition, ACTA Machanica Sinica, 1990, 6(4): 357

3 Liang, N.G., Liu, H.Q., Wang, T.C., A meso elastoplsatic constiturive model for folycysialline metals based on equivalent slip systems with latent hardening, Science in China, Ser, A, 1958, 41'8;: 33 ;.

4 Liu, H.Q., Hutter, K., On a ineso-elastıplastic constituaive eracition with application to deformation-induced anisotropy of a polycrystalline agorecsate, Arch. Mech, i; $96,4 i(1): j 3$.

5 Kalaml:ariv, A. L. . Liı, ił.Q., A new model for the multiphase fiber-matrix composite materials, Composites, 1998, 29B (5): $6: 3$

6 Kachanor L. M. , Time of the rupture process under creep condition, Ivz. Akad. Nauk. USSR. Otd. Tekhn Nauk (in Rus$\operatorname{sian),~1958,8:26.~}$

7 Housselier, G., Finite deformation constitutive relations including ductile fracture damage, in Proceedings of the IUTAM Symposium on Three-dimensional Constitutive Relations and Ductile Fracture, Dourdan, France, Amsterdam: North-Holland Pub. Company, 1980, 331-355.

8 Murakami, S., Notion of continuum damage mechanics and its application to anisotropic creep damage theory, ASME, J. Eng. Mat. and Tech., 1983, 105: 99.

9 Lemaitre, J., Chaboche, J.L., Aspect phenomenologique de la rupture par endommagement, J. Meca, Appl. (in French), $1978,2(3): 317$

10 Liu, H.Q., Liang, G. N., A physical-mechanism-based anisotropic damage-rate-dependent constitutive equation, in Second Asia-Pacific Symposium on Advances in Engineering Plasticity and its Application, Beijing: International Academic Publishers, $1994,155-160$

11 Krajcinovic, D., Damage Mechanics, Amsterdam: Elsevier Science B.V., 1996, Chap. 2.

$12 \mathrm{Xia}, \mathrm{M} . \mathrm{F} ., \mathrm{Ke}, \mathrm{F} . \mathrm{J}$., Bai,J. et al., Threshold diversity and trans-scales sensitivity in a finite nonlinear evolution model of materials failure, Phys. Lett. , Ser. A, 1997, 236: 60 .

13 Kuang, Z.B., Foundation of Nonlinear Continuum Mechanics (in Chinese), Xi' an: Xi' an Jiaotong University press, 1989, Chap. 4

14 Thomason, J. L., Vlug, M. A., Influence of fiber length and concentration on the properties of glass fiber-reinforced polypropylene 1: Tensile and flexural modulus, Composites, 1996, 27A(6): 477.

15 Thomason, J.L., Vlug, M. A., Schipper, G. et al., Influence of fiber length and concentration on the properties of glass fiber-reinforced polypropylene 3: Strength and strain at failure, Composites, 1996, 27A(6): 1075 . 